\title{
Endothelin Receptor Antagonists in Diabetic Nephropathy
}

\author{
René R Wenzel, ${ }^{1}$ Eberhard Ritz ${ }^{2}$ and Maximilian Q Wenzel ${ }^{3}$
}

1. Head, Department of Internal Medicine, Teaching Hospital, Zell am See;

2. Professor Emeritus, Ruperto Carola University Heidelberg; 3. Medical Student, University of Vienna

\begin{abstract}
Diabetic nephropathy (DN) is the leading cause of end-stage renal disease and affects an estimated 150 million people worldwide. Despite optimal treatment, including glycaemic control and antihypertensive therapy (e.g., renin-angiotensin-aldosterone system [RAS] blockade), the disease progresses. A 'late escape' phenomenon has been described, where proteinuria reappears despite continued RAS blockade. The endothelin (ET) system is strongly involved in the pathophysiology of the disease and contributes to vasoconstriction, inflammation and proliferation. ET antagonists are promising drugs that potently slow down disease progression in animal models and have beneficial effects on cardiac structure, mitochondrial damage and microvascular architecture. However, the available ET antagonists, at least in higher doses, may also inhibit tubular endothelin receptors subtype B, which promote sodium and water excretion. The three clinical trials with avosentan and atrasentan published so far show the unique nephroprotective effects of these drugs, with a reduction of up to $45 \%$ in albuminuria. However, fluid retention, oedema and, in higher stages of chronic kidney disease, heart failure limit their use. The reason may be that we have been using too high doses of these ET antagonists so far and they are inhibiting tubular sodium and water excretion. Thus, we will need to learn more about the role of ET and its antagonists in the tubular and collecting duct system, and on how to use these potent drugs in DN. ET antagonists are among the most promising molecules for the treatment of nephropathies. We should definitely not abandon these drugs because of the initial drawbacks in the first clinical trials.
\end{abstract}

\section{Keywords}

Endothelin antagonist, diabetic nephropathy, albuminuria, avosentan, atrasentan, renal failure, nephroprotection

Disclosure: René R Wenzel has received consultancy fees from Takeda, Menarini, Speedel Pharma, Actelion, Abbott and Novartis. Eberhard Ritz has received consultancy fees from Abbott, Daiichi Sankyo, Boehringer Ingelheim and Hexal. Maximilian Q Wenzel has no conflicts of interest to declare.

Received: 21 December 2011 Accepted: 16 January 2012 Citation: European Endocrinology, 2012;8(1):32-5 DOI:10.17925/EE.2012.08.01.32

Correspondence: René R Wenzel, Head, Department of Internal Medicine, Teaching Hospital of the Paracelsus University Salzburg, Paracelsusstr. 8, A-5700 Zell am See, Austria. E: rene@rrwenzel.at

Diabetic nephropathy (DN) was discovered by the British physician Clifford Wilson (1906-1997) and the German-born American physician Paul Kimmelstiel (1900-1970). Their first publication reporting the disease came out in 1936. ${ }^{1}$ Overt nephropathy secondary to glomerular disease usually occurs 15-25 years after the diagnosis of diabetes. In the past, it affected $25-35 \%$ of type 1 diabetic patients under the age of 30 years, but the prognosis of these patients has recently improved. ${ }^{2}$ The main issue today is type 2 diabetes. Approximately $2 \%$ of type 2 diabetic patients develop both macroalbuminuria and end-stage kidney disease. ${ }^{3}$ Nephropathy in type 2 diabetes has become the most common cause of chronic kidney failure and end-stage kidney disease in the world. Approximately $50 \%$ of patients admitted for renal replacement therapy have type 2 diabetes. The progressive increase seen in the past has stabilised in recent years at this high level. The basic problem is the high prevalence of type 2 diabetes in the developed world. The International Diabetes Federation predicts an $75 \%$ increase in the worldwide incidence of diabetes. This would mean that, by 2025 , 324 million people would be diabetic. Thus, the expected number of DN cases is huge.

Optimal treatment of diabetes, especially glycaemic and blood pressure control, have markedly improved renal prognosis in the past decade. Any antihypertensive therapy reduces renal damage and delays the decline of glomerular filtration rate (GFR). ${ }^{4}$

Due to the progressive nature of $\mathrm{DN}$, treating the disease is challenging. Current first-line therapies include lowering of blood pressure plus blockade of the renin-angiotensin-aldosterone system (RAS) with angiotensin converting enzyme (ACE)-inhibitors and/or angiotensin receptor blockers (ARBS). These treatments reduce proteinuria and delay the time to end-stage renal disease (ESRD) in patients with type 1 and type 2 diabetic nephropathy. ${ }^{4-8}$ However, the therapeutic efficacy of this approach is limited and suboptimal. ${ }^{9}$ Of particular concern is the phenomenon of 'late escape', i.e., the recurrence of proteinuria despite continued RAS blockade. ${ }^{5}$ But the most pressing problems are the incomplete reduction of proteinuria and the failure to completely block GFR loss, warranting additional interventional strategies. In this context, an activated endothelin (ET) system may contribute to the progression of DN. ${ }^{5}$

\section{Role of the Endothelin System in Diabetic Nephropathy}

ET was first described in 1988 by Yanagisawa et al. and is the most potent vasoconstrictor known. ${ }^{6}$ However, several other effects of ET have been discovered, including the stimulation of vascular and 
myocardial growth and inflammation. ${ }^{7.8}$ The initial stages of DN involve subtle morphologic changes in the renal glomeruli, with progression to microalbuminuria, macroalbuminuria and, ultimately, ESRD. ${ }^{?}$

The ET system plays an important role in the pathophysiology, not only of cardiovascular disease but also of renal disease..$^{8,10}$ ET-1 regulates a number of renal functions ${ }^{11}$ and causes proteinuria by several different mechanisms..$^{12,13}$ In the kidney, both the endothelin receptors subtype $A$ (ETAS) and the endothelin receptors subtype $B$ (ETBS) are present but differ in their their place and function: the ETA is mainly present in the renal vasculature, whereas the ETB predominates in the tubule-interstitium, the endothelium and the mesangium. ${ }^{14}$ ET-1 promotes growth and inflammation at the level of the kidney and regulates sodium and water retention as well as acid secretion (see below). It is interesting to note that mesangial cells can rapidly release ET-1 in response to injuries including hypoxia, hypertension and high glucose concentrations. ${ }^{15}$

In DN, the ET system is overactive, as indicated by the elevated plasma and urinary ET-1 levels found in patients..$^{16}$ Increased plasma ET-1 concentrations found in type 2 diabetes patients correlate with the severity and duration of diabetes. ${ }^{17,18}$ Importantly, in diabetic patients with nephropathy, raised levels of ET-1 also correlate with reduced renal function, increased blood pressure and albuminuria. ${ }^{18}$ Insulin increases renal ET expression, which may be especially relevant in diabetics with insulin resistance in an earlier phase of the disease when higher insulin concentrations occur. ${ }^{19}$

\section{Role of Endothelin Receptor Antagonists in Experimental Diabetic Nephropathy}

After the groundbreaking discovery of the endothelial-cell-derived vasopressor agent ET by Yanagisawa et al., inhibitors of the ET receptor were high on the priority list of the pharmaceutical industry. After various promising experimental trials, clinical studies in various pathologies with an assumed pathophysiological role of ET were conducted. ${ }^{20,21}$ Although several were disappointing, we increasingly learned from these trials - mainly from the side effects. In patients with heart failure, high doses of bosentan and darusentan were investigated. Although pulmonary and systemic haemodynamics were improved, the overall outcome was negative because of increases in morbidity and mortality, mainly due to volume overload. As a result, the treatment of cardiovascular disease with ET receptor antagonists was abandoned..$^{20,21}$ On the other hand, in one specific condition, i.e., pulmonary arterial hypertension, several clinical trials yielded positive results, leading to a well-defined indication for this class of drugs. ${ }^{22}$

Another attractive target was renal disease. The various renal effects of ET had led to the hypothesis that ET receptor antagonists were presumably renoprotective. ${ }^{16}$ Learning from the experiences in pulmonary arterial hypertension, and in contrast to congestive heart failure, many investigators were convinced that renoprotection was provided not by the haemodynamic effects of ET receptor antagonists, but rather by their anti-inflammatory and antiproliferative effects. This led to several studies with the goal of protecting the kidney in diabetic and non-diabetic nephropathies.

Various studies have shown marked beneficial effects of ET receptor antagonists in animal models of diabetes and, although these models are limited in terms of their comparability with the human kidney, they still helped us understand the mechanisms of these beneficial effects
Among a great number of experiments, two experimental studies which had led to the hypothesis of renoprotection by ET blockade will be discussed in more detail here. In the trial by Gagliardini et al., ${ }^{23}$ streptozotocin diabetic rats were uninephrectomised and treated with an ACE-inhibitor (lisinopril) and ETA-antagonist (avosentan), or both. Therapy with both lisinopril and avosentan normalised proteinuria, prevented tubulointerstitial damage and induced a regression of glomerular lesions. Interestingly, while only partial renoprotection was achieved by each drug alone, the two drugs, which both reduced proteinuria, affected different renal targets: lisinopril improved pathological glomerular size selectivity to large macromolecules, whereas avosentan ameliorated peritubular capillary architecture and reduced interstitial inflammation and fibrosis. ${ }^{23}$

In a recently published trial conducted by Zoja et al., Zucker diabetic fatty rats were treated orally with an ACE-inhibitor (ramipril), with an ETA-antagonist (sitaxsentan) or with both. ${ }^{24}$ In this setting, the antiproteinuric effects could mainly be attributed to the ACE-inhibitor. However, the ETA-antagonist had important effects on cardiac structure, mitochondrial damage and microvascular architecture, mainly through blocking the effects of VEGF-1.

Several other experimental trials have been published in the past decade, all of which suggest a nephroprotective effect of ET receptor antagonists alone and/or in combination with a blocker of the RAS. ${ }^{15}$ The question still remains whether such beneficial effects are mainly due and restricted to an antagonism at the level of the ETA. Indeed, stimulation of renal ETB may have beneficial effects via increased NO release and sodium excretion. Antagonists that block both ET receptors (so-called dual ET receptor antagonists) as well as very high doses of ET receptor antagonists with a lower selectivity for the ETA may therefore be disadvantageous. Unfortunately, the role of the ETB in kidney disease is unclear and it may counteract or contribute to renal damage. ${ }^{25}$

\section{Role of Endothelin Receptor Antagonists in Clinical Diabetic Nephropathy}

Encouraged by experimental data and by a promising pilot trial, we performed a multicentre study in more than 50 European centres (the SPEED II trial). ${ }^{26}$ The study population comprised 286 patients with DN (chronic kidney disease [CKD] stage II). The ETA-selective receptor antagonist avosentan was given at doses of 5, 10, 25 and $50 \mathrm{mg}$ on top of RAS blockade (with ACE-inhibitors or ARBS) for 12 weeks and compared with placebo. The trial was supported by Speedel Pharma AG (Basel, switzerland) and higher doses (i.e., 25 and $50 \mathrm{mg}$ ) were favoured, mainly based on haemodynamic effects and animal experiments. The primary endpoint, urinary albumin excretion rate (UAER), was dramatically reduced by up to $45 \%$. Unfortunately, at the higher doses of avosentan, oedema and fluid retention occurred in up to $26 \%$ of patients. Furthermore, no further antiproteinuric effect could be shown with avosentan doses above $10 \mathrm{mg}{ }^{26}$

A Phase III trial (the ASCEND study, ClinicalTrials.gov identifier NCT00120328) was performed, looking at more than 1,300 patients with $\mathrm{DN}$, but with a more advanced stage of kidney disease - i.e., CKD stage IIIb. ${ }^{27}$ Despite the side effects with higher doses known from our preceding trial, the ASCEND study investigated 25 and $50 \mathrm{mg}$ of avosentan over six months on top of RAS blockade and compared the effects with placebo. Again, there was a marked reduction in UAER of up to $49 \%$. After a median treatment period of four months, the 
Figure 1: Comparison of the Anti-albuminuric Effects (Panel A) and Side Effects (Panel B) of Endothelin Receptor Antagonists in the Three Published Clinical Trials in Diabetic Nephropathy ${ }^{26-28}$

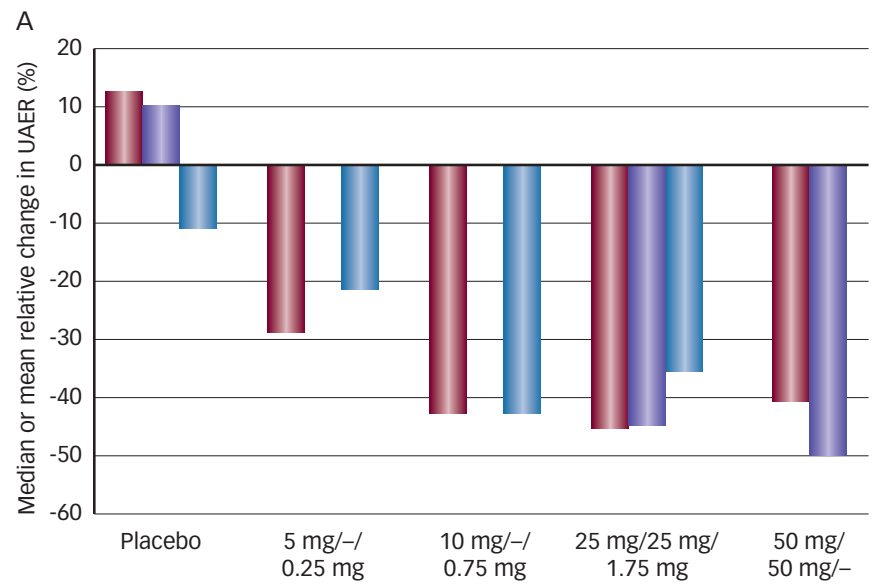

B

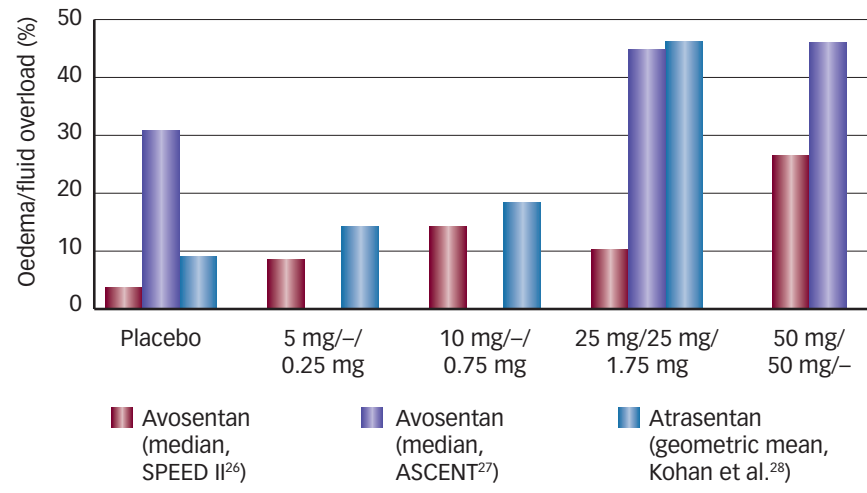

UAER = urinary albumin excretion rate. The $x$-axes show the respective doses of endothelin receptor antagonists (avosentan or atrasentan) studied in the three trials. ${ }^{26-28}$

Data Safety and Monitoring Board terminated the study prematurely, because of an excessive frequency of oedema, fluid overload and congestive heart failure. ${ }^{27}$

Finally, a recently published trial by Kohan et al. assessed the effects of the newly developed, more ETA-selective receptor antagonist atrasentan $\left(0.25,0.75\right.$ and $1.75 \mathrm{mg}$ ) in 89 patients with DN (CKD stage II-Illa). ${ }^{28}$ UAER was reduced by up to $35 \%$ compared with placebo. Peripheral oedema occurred in up to $46 \%$ of patients, mainly in the high doses.

In all three trials, GFR was unchanged after treatment with the respective ET receptor antagonist. ${ }^{26-28}$

\section{What Can We Learn from these Three Important Clinical Trials?}

There is no doubt that the ET receptor antagonists studied in the three trials - i.e., avosentan and atrasentan - dramatically reduce proteinuria and thus are likely to be highly nephroprotective. However, their use in this setting is currently still limited by their side effects - mainly fluid overload

When we compare the effects and side effects of the two drugs in the three trials, it is evident that lower doses of both avosentan and atrasentan are antiproteinuric, with a low rate, if any, of side effects. In contrast, with higher doses (i.e., 25 and 50 mg avosentan and
$1.75 \mathrm{mg}$ atrasentan), a significant rise in the frequency of side effects is seen without any relevant additive antiproteinuric effect (see Figure 1). ${ }^{26-28}$ Fluid overload becomes increasingly significant, especially in patients with advanced disease such as those studied in the ASCEND trial (CKD IIIb). Whether low dietary sodium and/or a adequately dosed diuretic regimen will be effective remains to be seen. We believe that these treatments are mandatory when any drug that causes fluid retention is given.

We know from experimental trials that ET promotes sodium excretion, mainly via ETBS at the level of the collecting duct. ${ }^{29}$ Consequently, when ET receptor antagonists are used that also block the ETB, at least partially, sodium retention will be a major concern. In fact, in healthy volunteers, the administration of avosentan for one week reduced sodium excretion and increased body weight for one to two days, but body weight subsequently normalised, presumably with the onset of compensatory mechanisms. ${ }^{30}$ Furthermore, it has been postulated that ET has a sodium-independent effect on vasopressin-dependent excretion of electrolyte-free water. ${ }^{29}$ While, in the atrasentan study by Kohan et al., the drug had no significant effect on body weight, the lowering of haemoglobin levels suggested an internal fluid shift - i.e., redistribution of fluid into the vascular compartment. ${ }^{28}$ In clinical practice, patients treated with ET receptor antagonists, particularly if their GFR is markedly reduced, should be advised to reduce sodium intake and should also be given adequate doses of diuretics - keeping in mind that, in proteinuric patients, the dose-response relationship is altered because of intratubular protein binding of diuretics. ${ }^{31}$

\section{Challenges for the Future - Effects and Side Effects of Endothelin Receptor Antagonists}

Today, progression of DN still occurs despite treatment according to guideline recommendations, including RAS blockade. ET receptor antagonists are one of the most promising classes of drugs on the horizon because of their antiproteinuric, anti-inflammatory and antiproliferative properties. The use of the currently available ET receptor antagonists is limited by their side effects, mainly the result of sodium-dependent and sodium-independent fluid retention leading to oedema and heart failure, particularly when administered at higher doses and in predisposed patients with higher stages of renal disease (i.e., CKD III or higher).

It is worthwhile looking beyond renoprotection. Even a minor reduction of renal function is associated with a dramatic increase in non-fatal and fatal cardiovascular events. In this context, again, ET receptor antagonists are of considerable interest: in the heart, they improve cardiac structure and microvascular architecture and reduce mitochondrial damage, and in the vasculature they reduce vascular remodelling and vessel wall hypertrophy, at least under experimental conditions..$^{24,32}$

Apart from their renoprotective properties, ET receptor antagonists will also be welcome because a significant proportion of patients with CKD also have cardiac problems and resistant hypertension, which will justify the use of ET receptor antagonists as additional antihypertensive and (potentially) somewhat cardioprotective agents. ${ }^{33}$

In many discussions, the following issues have been raised: should ET receptor antagonists be abandoned because of their side effects? Do the potential problems with fluid overload negate the beneficial 
effects on renal and myocardial tissue? Would more selective ET receptor antagonists with a higher affinity for the ETA be equally renoprotective but induce fewer side effects?

Although we do not have the evidence-based answers to all these questions, the overwhelmingly positive findings in experimental studies are promising and should encourage the continuation of well-designed trials that take into consideration the selection of the right dose and of an appropriately subclass-specific ET receptor antagonist. Such studies should be performed in well-defined populations, excluding patients with major pre-existing cardiac problems (mainly heart failure). The study protocols should include advice on how to achieve low dietary sodium intake, possibly the co-administration of diuretics that are active in the collecting duct, and a strict weight-gain control. Beyond such indispensable clinical studies, we also need additional experimental data on the role of ET-receptor subtypes in the kidney, especially in the collecting duct. ${ }^{31}$

We are convinced that, in future, ET receptor antagonists will have a role to play in patients with renal and reno-cardiac disease. Predictably, the benefit will be greatest if these drugs are used at earlier stages of renal disease than in the above studies, mainly because, at these earlier stage, fluid retention is less of a problem. Betablockers, once contraindicated in heart failure patients because of their side effects, are now the cornerstone of therapy. Maybe one day ET receptor antagonists will know the same fate?
1. Kimmelstiel $\mathrm{P}$, Wilson $\mathrm{C}$, Benign and malignant hypertension and nephrosclerosis: a clinical and pathological study, Am J Pathol, 1936;12:45-82.3

2. Orchard TJ, Dorman JS, Maser RE, et al., Prevalence of complications in IDDM by sex and duration. Pittsburgh Epidemiology of Diabetes Complications Study II, Diabetes, 1990;39:1116-24.

3. Adler Al, Stevens RJ, Manley SE, et al., Development and progression of nephropathy in type 2 diabetes: the United
Kingdom Prospective Diabetes Study (UKPDS 64), Kidney Int, Kingdom Prospectiv

4. Wenzel RR, Renal protection in hypertensive patients: selection of antihypertensive therapy, Drugs, 2005;65(Suppl. 2):29-39.

5. Wühl E, Trivelli A, Picca S, et al., Strict blood-pressure contro and progression of renal failure in children, New Eng $J$ Med, 2009;361:1639-50

6. Yanagisawa M, Kurihara H, Kimura S, et al., A novel potent vasoconstrictor peptide produced by vascular endothelia cells, Nature, 1988:332:411-5.

7. Wenzel RR, Zbinden $\mathrm{S}$, Noll G, et al., Endothelin-1 induces vasodilation in human skin by nociceptor fibres and release of nitric oxide, Br J Clin Pharmacol, 1998;45:441-6.

8. Wenzel RR, Czyborra P, Lüscher T, Philipp T, Endothelin in cardiovascular control: the role of endothelin antagonists, Curr Hypertens Rep, 1999;1:79-87.

9. Adler Al, Managing diabetes: what to do about cardiovascular disease, Diabetes Res Clin Pract, 2003;61(Suppl. 1):S3-8.

10. Wenzel RR, Role of angiotensin-1-receptor blockers in cardiorenal disease, Current Drug Therapy, 2006;1:47-54.

11. Rubanyi GM, Polokoff MA, Endothelins: molecular biology, biochemistry, pharmacology, physiology, and pathophysiology, Pharmacol Rev, 1994:46:325-415.

12. Chen S, Evans T, Deng D, et al., Hyperhexosemia induced functional and structural changes in the kidneys: role of endothelins, Nephron, 2002;90:86-94.
13. Sorokin A, Kohan DE, Physiology and pathology of endothelin-1 in renal mesangium, Am J Physiol Renal Physiol, 2003;285:F579-89.

14. Rebibou JM, He CJ, Delarue F, et al., Functional endothelin receptors on human glomerular podocytes and mesangial cells, Nephrol Dial Transplant, 1992;7:288-92.

15. Benz K, Amann K, Endothelin in diabetic renal disease Contrib Nephrol, 2011:172:139-48.

16. Wenzel RR, Czyborra P, Luscher T, Philipp T, Endothelin in cardiovascular control: the role of endothelin antagonists, Curr Hypertens Rep, 1999:1:79-87.

17. Letizia C, Iannaccone A, Cerci S, et al., Circulating endothelin-1 in non-insulin-dependent diabetic patients with retinopathy, Horm Metab Res, 1997;29:247-51.

18. Peppa-Patrikiou M, Dracopoulou M, Dacou-Voutetakis C, Urinary endothelin in adolescents and young adults with insulin-dependent diabetes mellitus: relation to urinary albumin, blood pressure, and other factors, Metabolism, 1998;47:1408-12.

19. Hargrove GM, Dufresne J, Whiteside C, et al., Diabetes mellitus increases endothelin-1 gene transcription in rat kidney, Kidney Int, 2000;58:1534-45.

20. Packer M, McMurray J, Massie BM, et al., Clinical effects of endothelin receptor antagonism with bosentan in patients with severe chronic heart failure: results of a pilot study, I Card Fail, 2005;11:12-20.

21. Kelland NF, Webb DJ, Clinical trials of endothelin antagonists in heart failure: a question of dose? Exp Biol Med (Mawwood) 2006;231:696-9

22. Barst RJ, Gibbs IS, Ghofrani HA, et al., Updated evidencebased treatment algorithm in pulmonary arterial hypertension, J Am Coll Cardiol, 2009:54:S78-84.

23. Gagliardini E, Corna D, Zoja C, et al., Unlike each drug alone lisinopril if combined with avosentan promotes regression of renal lesions in experimental diabetes, Am J Physiol Renal Physiol, 2009;297:F1448-56
24. Zoja C, Cattaneo S, Fiordaliso $\mathrm{F}$, et al. Distinct cardiac and renal effects of ETA receptor antagonist and ACE inhibitor in experimental type 2 diabetes, Am J Physiol Renal Physiol, 2011;301:F1114-23

25. Ritz $\mathrm{E}$, Wenzel $\mathrm{R}$, Endothelin receptor antagonists in proteinuric renal disease: every rose has its thorn, J Am SOC Nephrol, 2010;21:392-4.

26. Wenzel RR, Littke T, Kuranoff S, et al., SPP301 (Avosentan) Endothelin Antagonist Evaluation in Diabetic Nephropathy Study Investigators, Avosentan reduces albumin excretion in diabetics with macroalbuminuria, I Am Soc Nephrol 2009:20:655-64.

27. Mann JF, Green D, Jamerson K, et al., ASCEND Study Group Avosentan for overt diabetic nephropathy, J Am Soc Nephrol, 2010;21:527-35

28. Kohan DE, Pritchett $Y$, Molitch $M$, et al., Addition of atrasentan to renin-angiotensin system blockade reduces albuminuria in diabetic nephropathy, J Am Soc Nephrol, 2011;22:763-72.

29. Kohan DE, Rossi NF, Inscho EW, Pollock DM, Regulation of blood pressure and salt homeostasis by endothelin, Physio Rev, 2011;91(1):1-77.

30. Smolander J, Vogt B, Maillard M, et al., Dose-dependen acute and sustained renal effects of the endothelin receptor antagonist avosentan in healthy subjects, Clin Pharmacol Ther, 2009;85:628-34.

31. Ritz E, Wenzel RR, Endothelin antagonist as add-on treatment for proteinuria in diabetic nephropathy: is there light at the end of the tunnel? J Am Soc Nephrol, 2011;22:593-5.

32. Moreau $\mathrm{P}$, Takase $\mathrm{H}$, Kung $\mathrm{CF}$, et al., Blood pressure and vascular effects of endothelin blockade in chronic nitric oxide-deficient hypertension, Hypertension, 1997:29:763-9.

33. Weber MA, Black $H$, Bakris $G$, et al., A selective endothelinreceptor antagonist to reduce blood pressure in patients with treatment-resistant hypertension: a randomised, double-blind placebo-controlled trial, Lancet, 2009;374:1423-31. 